\title{
Mechanism of Hydration and Hydration Induced Structural Changes of Calcium Alginate Aerogel
}

\author{
Attila Forgács ${ }^{\mathrm{a}, \mathrm{b}}$, Vanda Papp ${ }^{\mathrm{a}}$, Geo Paul ${ }^{\mathrm{c}}$, Leonardo Marchese ${ }^{\mathrm{c}}$, Adél Len ${ }^{\mathrm{d}}$, \\ Zoltán Dudás ${ }^{\mathrm{d}}$, István Fábián ${ }^{\mathrm{a}, \mathrm{b}}$, Pavel Gurikove and József Kalmár ${ }^{\mathrm{a}, \mathrm{b}, *}$
}

a Department of Inorganic and Analytical Chemistry, University of Debrecen, Egyetem tér 1, Debrecen, H-4032 Hungary

${ }^{\mathrm{b}}$ MTA-DE Redox and Homogeneous Catalytic Reaction Mechanisms Research Group, Egyetem tér 1, Debrecen, H-4032 Hungary

${ }^{c}$ Department of Science and Technological Innovation, Universitá del Piemonte Orientale, Viale T. Michel 11, 15121 Alessandria, Italy

${ }^{\mathrm{d}}$ Neutron Spectroscopy Department, Centre for Energy Research, Konkoly-Thege Miklós út 29-33, Budapest, H-1121 Hungary

${ }^{\mathrm{e}}$ Laboratory for Development and Modelling of Novel Nanoporous Materials, Hamburg University of Technology, Eißendorfer Straße 38, 21073 Hamburg, Germany

* Corresponding author: e-mail: kalmar.jozsef@science.unideb.hu 


\section{Chemicals and preparation of Ca-alginate aerogel (CaAG) beads}

Sodium alginate was purchased from Sigma life science (catalogue no. 71238). Ethanol (99.8) and $\mathrm{CaCl}_{2}$ were purchased from H. Möller GmbH (Germany) and Th. Geyer GmbH \& Co. KG, Lohmar, Germany, respectively, and used without any further purification. Double deionized and ultrafiltered water $(\rho=18.2 \mathrm{M} \Omega \mathrm{cm}$ ) prepared by a MilliQ (Millipore) equipment was used for all experiments. Carbon dioxide with a purity of $>99.9 \%$ was supplied by AGA Gas GmbH (Hamburg, Germany).

Spherical Ca-alginate aerogel $(\mathrm{CaAG})$ beads of ca. $0.2 \mathrm{~mm}$ were synthesized using the jet cutting method as described previously. ${ }^{1-2}$ Briefly, $1 \mathrm{w} / \mathrm{w} \%$ aqueous solution of Na-alginate was dropped into $\mathrm{CaCl}_{2}$ solution $(20 \mathrm{~g} / \mathrm{L})$ using a commercial JetCutter (Type $\mathrm{S}$ from geniaLab $\mathrm{GmbH}$, Braunschweig, Germany). The gelation bath was moderately stirred in order to ensure the formation of spherical beads and to prevent their aggregation.

After gelation, the gel beads were subjected to multiple step solvent exchange. The Caalginate beads were placed for $24 \mathrm{~h}$ into 30,60 and $90 \mathrm{w} / \mathrm{w} \%$ ethanol-water mixtures and then two times into anhydrous ethanol. Hydrogel-to-solution volume ratio was at least 5. After the last step, the ethanol content in the soaking liquid was checked by measuring its density with a density meter (DMA 4500, Anton Paar Company, Austria). The drying procedure was initiated only when the ethanol content reached $\min .98 .5 \mathrm{w} / \mathrm{w} \%$ in order to ensure single phase conditions during subsequent supercritical drying.

The gel beads were dried by extraction of ethanol with a continuous flow of supercritical $\mathrm{CO}_{2}$ in a high-pressure autoclave. Briefly, filter paper bags containing the samples were placed into a high-pressure autoclave $(250 \mathrm{~mL})$ and covered with a few milliliters of ethanol in order to prevent premature solvent evaporation. The autoclave was sealed and preheated to $313-$ 323 K. Preheated carbon dioxide was supplied into the autoclave until the desired working pressure (12 MPa) was achieved. Then, the outlet valve was adjusted to achieve carbon dioxide 
flow rate through the autoclave of ca. $25 \mathrm{~g} \mathrm{~min}^{-1}$. The outgoing flow was directed into a separator to split the fluid into ethanol and gaseous $\mathrm{CO}_{2}$. The drying lasted for $3 \mathrm{~h}$; then, the autoclave was slowly depressurized $\left(0.5 \mathrm{MPa} \mathrm{min}^{-1}\right)$ and the dry as-prepared CaAG beads were collected. The aerogel samples were kept in a desiccator under freshly dried silica gel. 


\section{Magic angle spinning (MAS) NMR spectroscopy}

Magic angle spinning (MAS) nuclear magnetic resonance (NMR) spectroscopy is able to effectively explore the structural evolution of polysaccharide aerogels upon hydration by following the ${ }^{13} \mathrm{C}$ and ${ }^{1} \mathrm{H}$ MAS NMR chemical shifts and linewidths. This technique provides information on the chemical composition of the backbone of the aerogel, the primary to quaternary structures of the macromolecular chains, the state of hydrogen bonding in the solid network, and the state and the localization of water in the hydrated sample. ${ }^{3-7}$ Importantly, the hydration of linear chain polysaccharides may cause alterations in the conformations of the chains or even the separation of chains that were originally bound together in the dry state of the material, that is well-expressed in the MAS NMR spectra. ${ }^{8}$

(M)

(M)

(G)

(G)

(G)

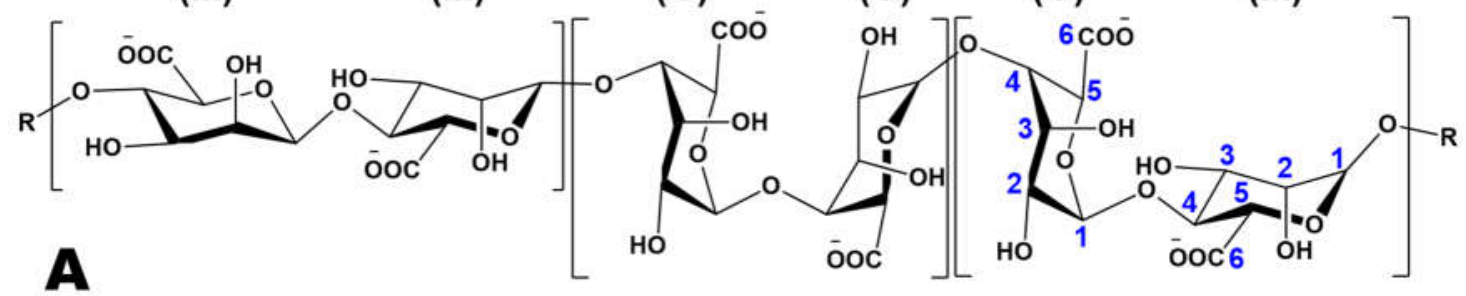

B

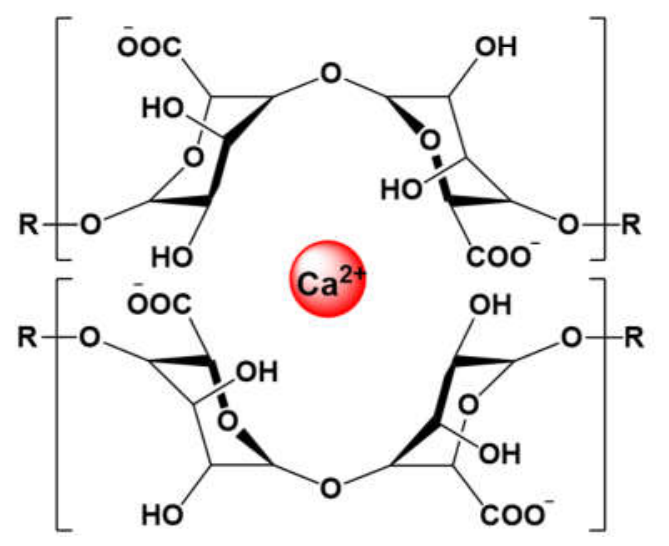

Figure S1. General molecular structure of alginate macromolecules (A), and the structure of the "eggbox" junction zone of the $\mathrm{Ca}^{2+}$ crosslinked alginate chains (B). $\mathrm{M}=\beta$-D-mannuronate unit, $\mathrm{G}=\alpha$-L-guluronate unit. 
Table S1. ${ }^{13} \mathrm{C}$ CPMAS NMR chemical shifts of the Ca-alginate aerogel assigned according to the spectrum of the hydrated aerogel of $1.0 \mathrm{~g} / \mathrm{g}$ water content. The nomenclature of the carbon atoms of Ca-alginate is given in Fig. S1. $\mathrm{M}=\beta$-D-mannuronate unit, $\mathrm{G}=\alpha$-L-guluronate unit.

\begin{tabular}{|l|c|}
\hline Carbons & ${ }^{13}$ C chemical shifts (ppm) \\
\hline G1, M1 & $101 ; 99$ \\
\hline G2, M2 & $65 ; 71$ \\
\hline G3, M3 & $68 ; 71$ \\
\hline G4, M4 & $81 ; 76$ \\
\hline G5, M5 & $68 ; 76$ \\
\hline G6, M6 & $175.5 ; 175.5$ \\
\hline
\end{tabular}

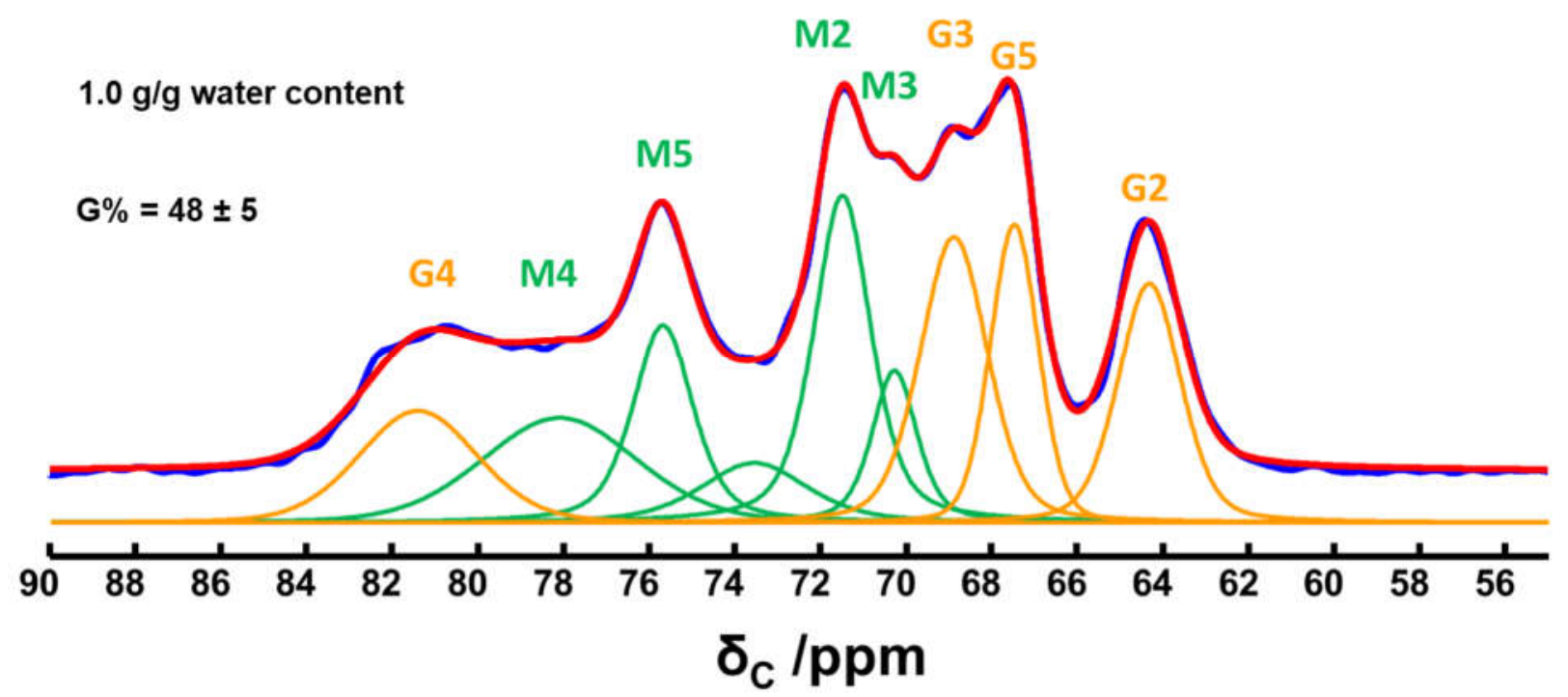

Figure S2. The deconvolution of the ${ }^{13} \mathrm{C}$ CPMAS NMR spectrum of the Ca-alginate of $1.0 \mathrm{~g} / \mathrm{g}$ water content. Based on the deconvolution, the percentage of the $\mathrm{G}$ units in the alginate chain is $48 \pm 5 \%$. The nomenclature of the carbon atoms of Ca-alginate is given in Fig. S1. $M=\beta$ D-mannuronate unit, $\mathrm{G}=\alpha$-L-guluronate unit. 


\section{Scanning electron microscopy (SEM)}

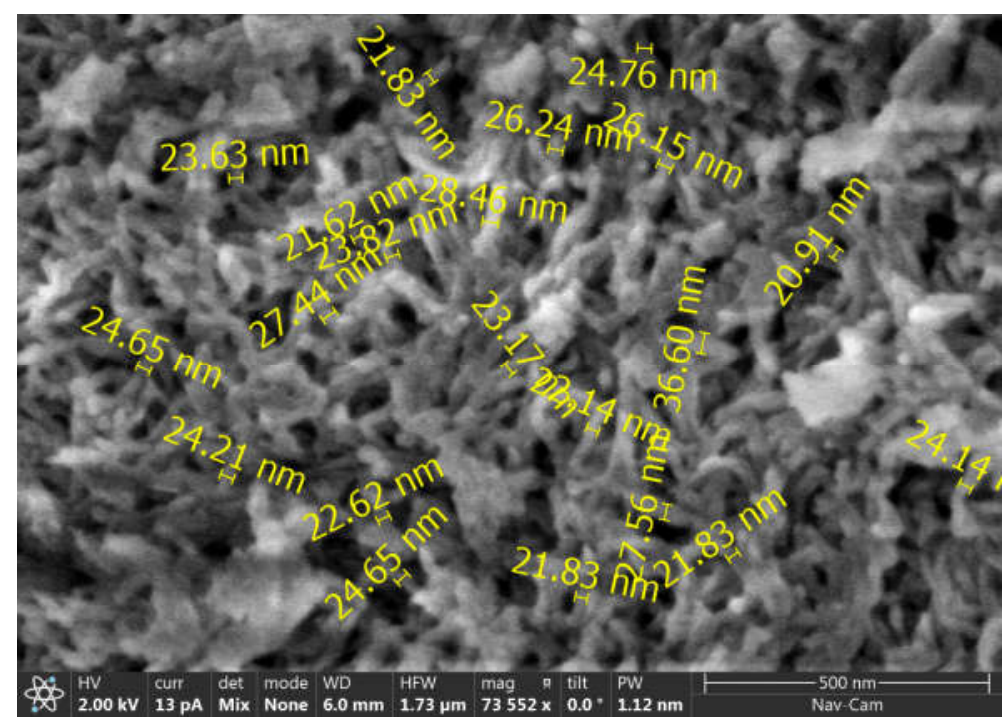

Figure S3. Scanning electron microscopy (SEM) image of CaAG showing the thickness of the primary fibrils.

\section{Nitrogen adsorption-desorption porosimetry}
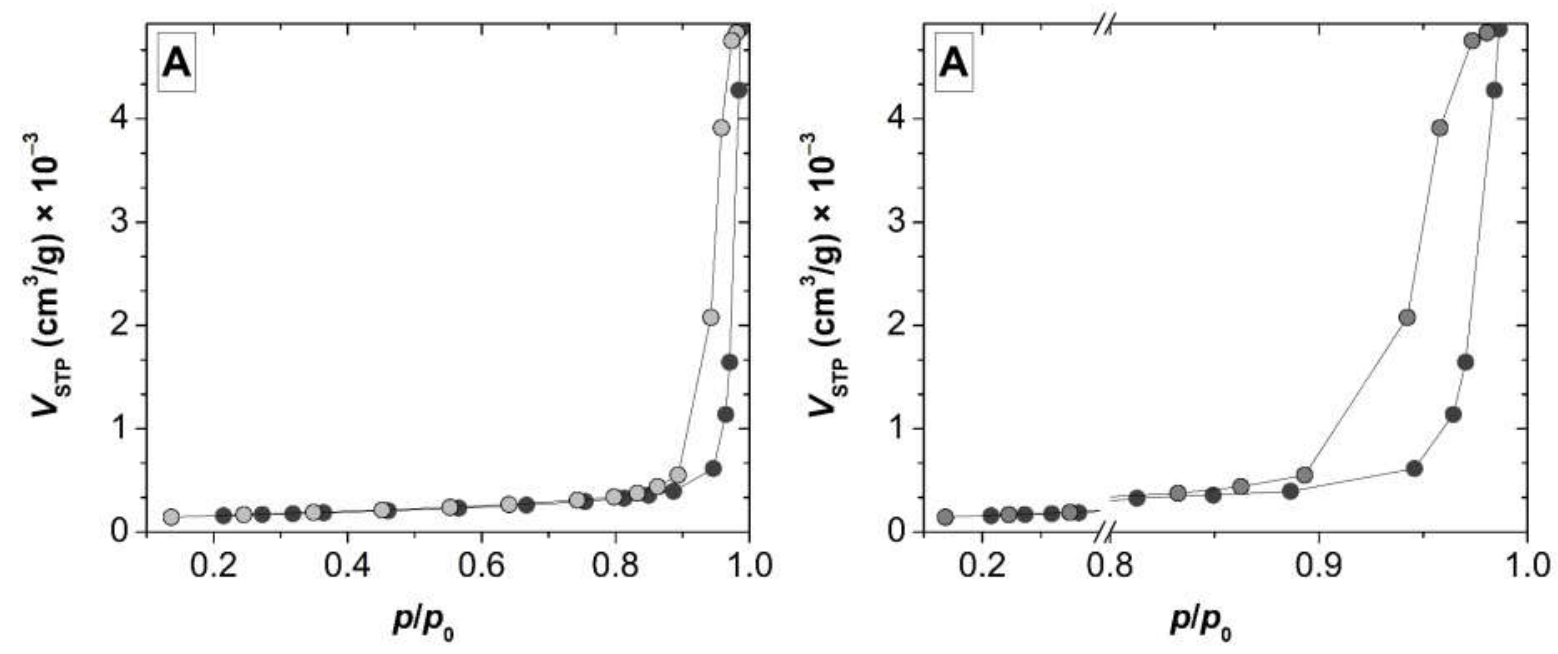

Figure S4. Nitrogen adsorption-desorption isotherms of as-prepared CaAG. Panel A: Isotherms in the full $p / p_{0}$ scale. Panel B: Magnification of the hysteresis loop. The data points are the same in the two panels. The isotherms are classified as type IV according to the IUPAC recommendation with a hysteresis loop of type H3. There is no steep rise in the isotherms at around $p / p_{0}=1$, indicating that there are practically no macropores in the aerogel. 


\section{Small angle neutron scattering (SANS)}

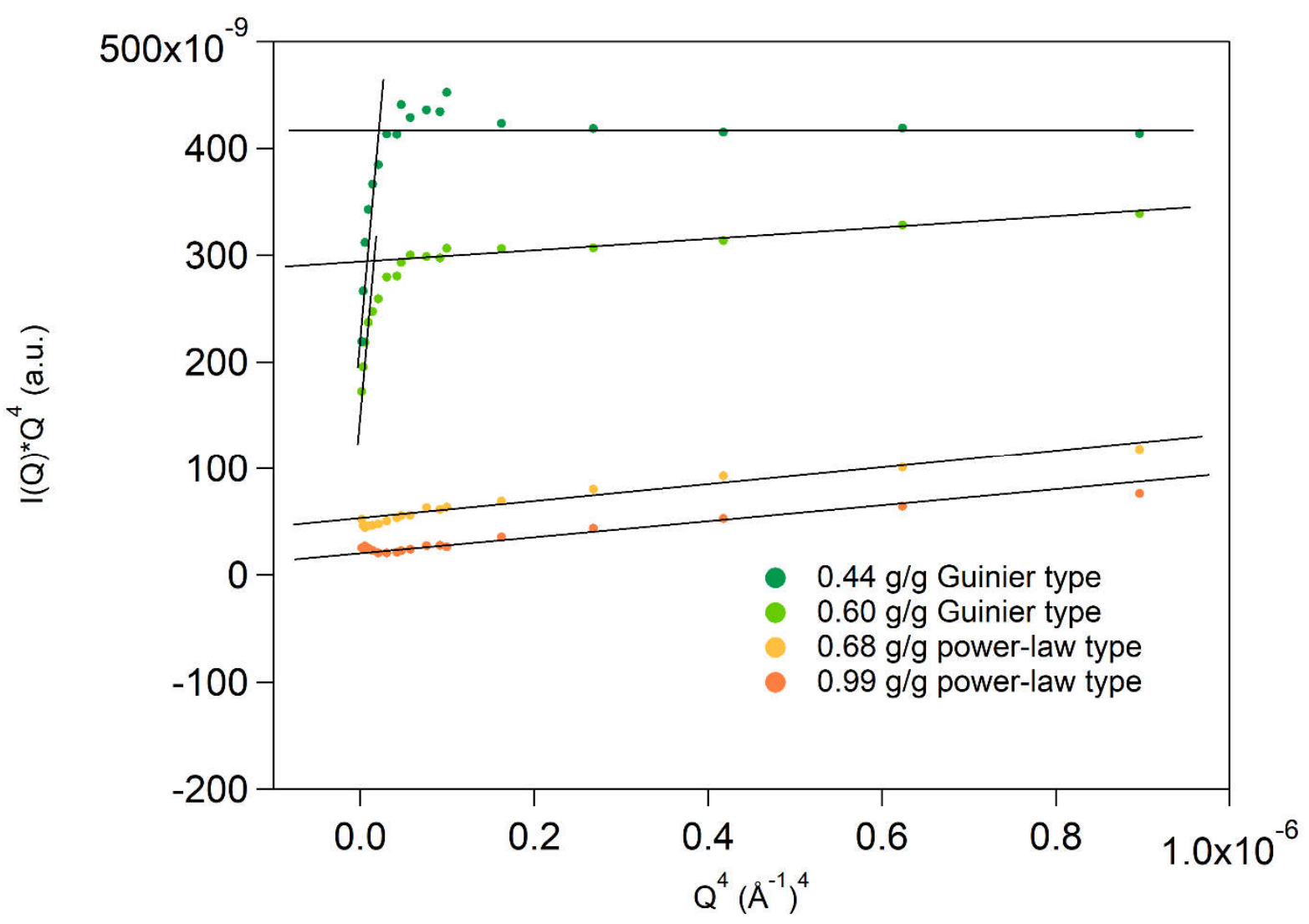

Figure S5. The Porod plot of SANS curves from Fig. 3 mesured at water contents near the transition point of $0.6 \mathrm{~g} / \mathrm{g}$. Water content is shown in the legend in $\mathrm{g} / \mathrm{g}$ units. 
Small angle neutron scattering (SANS)
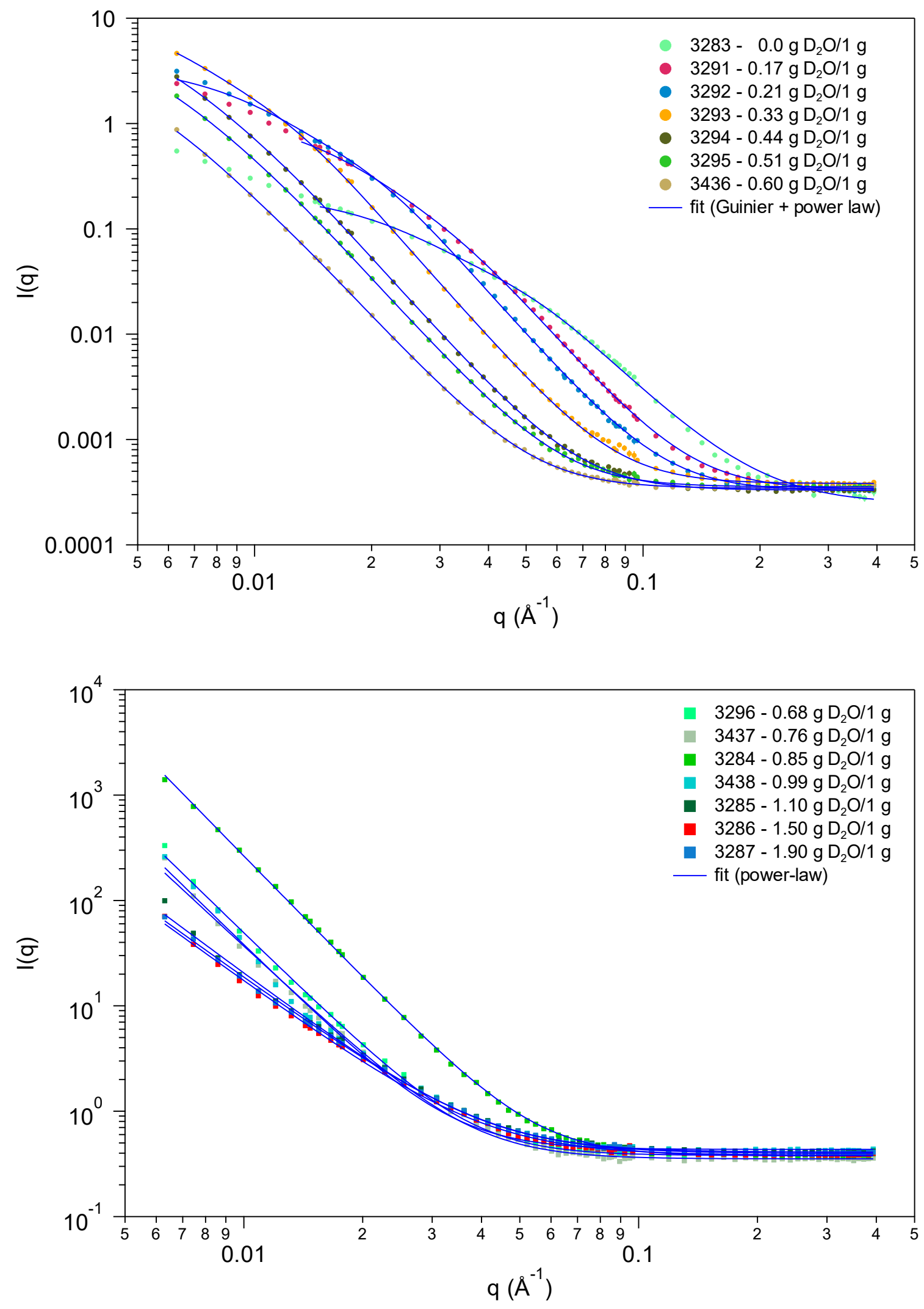

Figure S6. The results of the fitting of the SANS curves from Fig. 3 with the Beaucage model and the power-law model. 


\section{NMR relaxometry theory - Dependence of relaxation time on water content}

The relaxation time characteristic for a single domain is the combination of the different relaxation times of the water molecules located in the different micro-environments of the domain participating in the fast exchange of water among the micro-environments. The characteristic relaxation time of the domain $\left(T_{2}\right)$ can be expressed by the following equation in the simplest case, when water in the bulk of a quasi-spherical droplet is in fast exchange with the adjacent hydration sphere of the backbone: ${ }^{9-11}$

$$
\frac{1}{T_{2}}=\frac{V_{\mathrm{s}}}{V_{0}} \times \frac{1}{T_{2 \mathrm{~s}}}+\frac{V_{\mathrm{b}}}{V_{0}} \times \frac{1}{T_{2 \mathrm{~b}}}
$$

Here, $V_{0}$ is the total volume of water in the domain, $V_{\mathrm{s}}$ is the volume of water on the surface of the droplet that is part of the hydration sphere of the backbone, and $V_{\mathrm{b}}$ is the volume of water in the bulk of the droplet $\left(V_{0}=V_{\mathrm{s}}+V_{\mathrm{b}}\right) . T_{2 \mathrm{~s}}$ is the relaxation time of water in the hydration sphere, $T_{2 \mathrm{~b}}$ is the relaxation time of bulk water and $T_{2}$ is the observed relaxation time of the domain. Naturally, $T_{2 \mathrm{~s}}$ is significantly smaller than $T_{2 \mathrm{~b}}$. In the case when the relaxation domain consists of a quasi-spherical water droplet in exchange with the adjacent hydration sphere of the backbone, the observed relaxation time $\left(T_{2}\right)$ increases with the increasing water content of the domain $\left(V_{0}\right)$ if the droplet size increases. When the size of the droplet increases, the ratio of bulk water $\left(V_{\mathrm{b}}\right)$ over surface water $\left(V_{\mathrm{s}}\right)$ also increases, which in turn results in the increase of the observed $T_{2}$ according to eq. S1. 


\section{NMR cryoporometry theory}

The Carr-Purcell-Meiboom-Gill (CPMG) spin-echo pulse sequence was applied to eliminate the broad ${ }^{1} \mathrm{H}$ signal of the solid phase during the echo time. The remaining signal intensity is proportional to the amount of liquid water confined in the porous system, which changes gradually with temperature, as water freezes or ice melts. The melting and freezing points of liquids confined in nanometer wide spaces are lowered, as given by the modified Gibbs-Thomson equations:

$$
\begin{aligned}
& \Delta T_{\mathrm{m}}=T_{\mathrm{m}}-T_{0}=-\frac{n_{\mathrm{m}} K_{\mathrm{c}}}{r_{\mathrm{p}}} \\
& \Delta T_{\mathrm{f}}=T_{\mathrm{f}}-T_{0}=-\frac{n_{\mathrm{f}} K_{\mathrm{c}}}{r_{\mathrm{p}}}
\end{aligned}
$$

In eq. $\mathrm{S} 2, \Delta T_{\mathrm{m}}$ is the melting point depression expressed as a difference between the phase transition temperature of the bulk $\left(T_{0}\right)$ and the confined liquid $\left(T_{\mathrm{m}}\right) . K_{\mathrm{c}}$ is the cryoporometric constant, $n_{\mathrm{m}}$ is the geometric factor describing melting and $r_{\mathrm{p}}$ stands for the average pore radius. In eq. $\mathrm{S} 3$, the symbols denote the corresponding parameters for freezing. The values of $\Delta T_{\mathrm{m}}$ and $\Delta T_{\mathrm{f}}$, and $n_{\mathrm{m}}$ and $n_{\mathrm{f}}$ are different, because melting and freezing of liquids in confined spaces show a hysteresis in general. ${ }^{12-14}$

The pore size distribution of the aerogel was calculated using the methodology of Petrov and Furó. The measured water peak integrals were plotted against the temperature. Intensity versus temperature data were transformed to intensity versus pore size data using the modified Gibbs-Thomson equations (eqs. S2 and S3). The numerical derivation of these yields pore size distribution plots.

\section{Results of NMR cryoporometry}

The primary experimental data in NMR cryoporometry are the intensity of the ${ }^{1} \mathrm{H}$ NMR signal of water protons as a function of the temperature of the hydrated porous sample. The 
spin-echo type CPMG pulse sequence that is used to measure the ${ }^{1} \mathrm{H}$ NMR spectra contains a relaxation delay long enough to allow the complete transversal relaxation of the water molecules that are in the solid phase. Consequently, only those molecules contribute to the ${ }^{1} \mathrm{H}$ NMR signal that are not in the frozen phase and not in strong association with the solid backbone. ${ }^{12-15}$ Liquid water localized in water droplets filling the pores of the material or forming puddles in the focal points of the porous network have high $T_{2}$ relaxation times and pass the relaxation filter. The high curvature of these water droplets and puddles confined in the backbone results in a shift of the melting temperature (and the freezing temperature) of water. The phase transition temperature shifts are in quantitative relation with the shape and the size of the droplets and puddles, as described by the Gibbs-Thomson equations (eqs. S2, S3).

The correct interpretation of the NMR cryoporometry data is possible only by taking into account the structural information deduced from the SANS and the NMR relaxometry measurements. Importantly, hydrated CaAG retains its well-defined porous structure at $0.3 \mathrm{~g} / \mathrm{g}$ water content. Based on the NMR relaxometry results, water can be localized in the aerogel in 3 different structural regions at this hydration level: $i$ ) structural water associated to the Caalginate macromolecules $\left(1^{\text {st }}\right.$ relaxation domain $\left.), i i\right)$ in the primary hydration sphere $\left(2^{\text {nd }}\right.$ relaxation domain), and iii) in small droplets and puddles confined in the focal points of the porous network ( $3^{\text {rd }}$ relaxation domain). The $T_{2}$ relaxation time of the water molecules in the $1^{\text {st }}$ domain is too small to pass the relaxation filter of the pulse sequence of the NMR cryoporometry measurements. Water in the $2^{\text {nd }}$ relaxation domain has long enough $T_{2}$ to pass the relaxation filter, but ice formation is not feasible in the primary hydration sphere due to the strong interaction of water with the highly curved solid backbone. Therefore, the limiting low value of the ${ }^{1} \mathrm{H}$ NMR signal intensity detected at $256 \mathrm{~K}$ in the cryoporometry measurements is assigned to water in the primary hydration sphere. Water in droplets confined in the focal points of the aerogel network freezes and melts between $256 \mathrm{~K}$ and $273 \mathrm{~K}$, thus, the well-defined 
hysteresis of the ${ }^{1} \mathrm{H}$ NMR signal in Fig. 5A is assigned to the phase transformations of water in these droplets.

The minor discrepancy in the trend of the experimental points of the freezing curve at ca. $270 \mathrm{~K}$ is attributed to the local undercooling of water in some of the droplets in the sample. 


\section{Pulse Gradient Stimulated Echo (PGSTE) NMR diffusiometry theory}

A stimulated echo sequence was used featuring bipolar gradient pulses, longitudinal eddy current delay and two spoiler gradients. Here, the single gradient pulses of the PGSTE sequence are split into a pair of two pulses with opposite signs enclosing a $\pi$ pulse. The time between the two $\pi$ pulses is the observation time of the diffusion experiment $(\Delta)$. In the PGSTE sequence, magnetization is longitudinal ( $T_{1}$ decay) during most of the diffusion time. The total time the magnetization remains in transversal direction ( $T_{2}$ decay) is twice the period between the first two $\pi / 2$ pulses, which is approximately equivalent to the length of the gradient pulse $(\delta)$. One lobe of the bipolar gradient pair is $\delta / 2$. One lobe of the bipolar gradient pair is $\delta / 2$.

The observation time of the diffusion experiments $(\Delta)$ was varied between 8 and 130 ms. The length of the gradient pulse $(\delta)$ changed from 2 to $6 \mathrm{~ms}$. The pulsed gradient strength $(G)$ increased in 32 square distant steps from 0 to ca. $50 \mathrm{G} / \mathrm{cm}$. The diffusion data were evaluated according to the general expression: ${ }^{16-17}$

$I=I_{0} \exp \left\{-D_{\mathrm{obs}} \gamma^{2}(\Delta-\delta / 3) \delta^{2} G^{2}\right\}$

The coefficient $D_{\mathrm{obs}} \gamma^{2}$ was calculated for each experiment by fitting the exponential curve of eq. S4 to the measured echo intensity $(I)$ as a function of $G^{2}$, and using the known parameters. The real diffusion coefficient $\left(D_{\mathrm{obs}}\right)$ was calculated after calibration, which was based on measuring the diffusion of $\mathrm{D}_{2} \mathrm{O}$ in water by this method. When multiple diffusion domains are present in the sample, each domain is represented by a different single-exponential

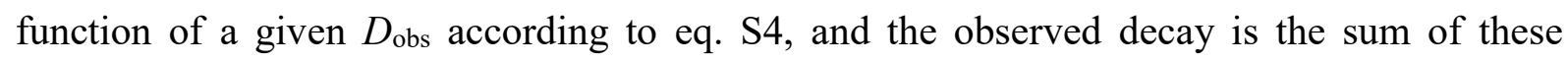
functions. If the exchange of water is slow between these domains, the different $D_{\text {obs }}$ values can be separated. ${ }^{18-20}$ In order to determine the number of diffusion domains, primary data were transformed by inverse Laplace transformation using the same algorithms as for the evaluation of NMR relaxometry data. 


\section{Results of PGSTE NMR diffusiometry}

It should be noted, that the results of the PGSTE measurements cannot be interpreted correctly using exclusively the conventional approaches of data evaluation of NMR diffusiometry. This is evident by examining the NMR spectra of the hydrated CaAG samples.

Figure S7 shows the ${ }^{1} \mathrm{H}$ NMR spectra of CaAG samples of different water contents next to their PGSTE NMR spectra recorded using $\delta=2 \mathrm{~ms}$ gradient pulse length and $\Delta=60 \mathrm{~ms}$ diffusion time. Two peaks can be identified in the ${ }^{1} \mathrm{H}$ NMR spectrum of CaAG of $0.2 \mathrm{~g} / \mathrm{g}$ water content, and this is replaced by one dominant peak in the case of the samples of higher hydration levels. The PGSTE NMR spectrum of the aerogel of $0.2 \mathrm{~g} / \mathrm{g}$ water content also displays 2 peaks.
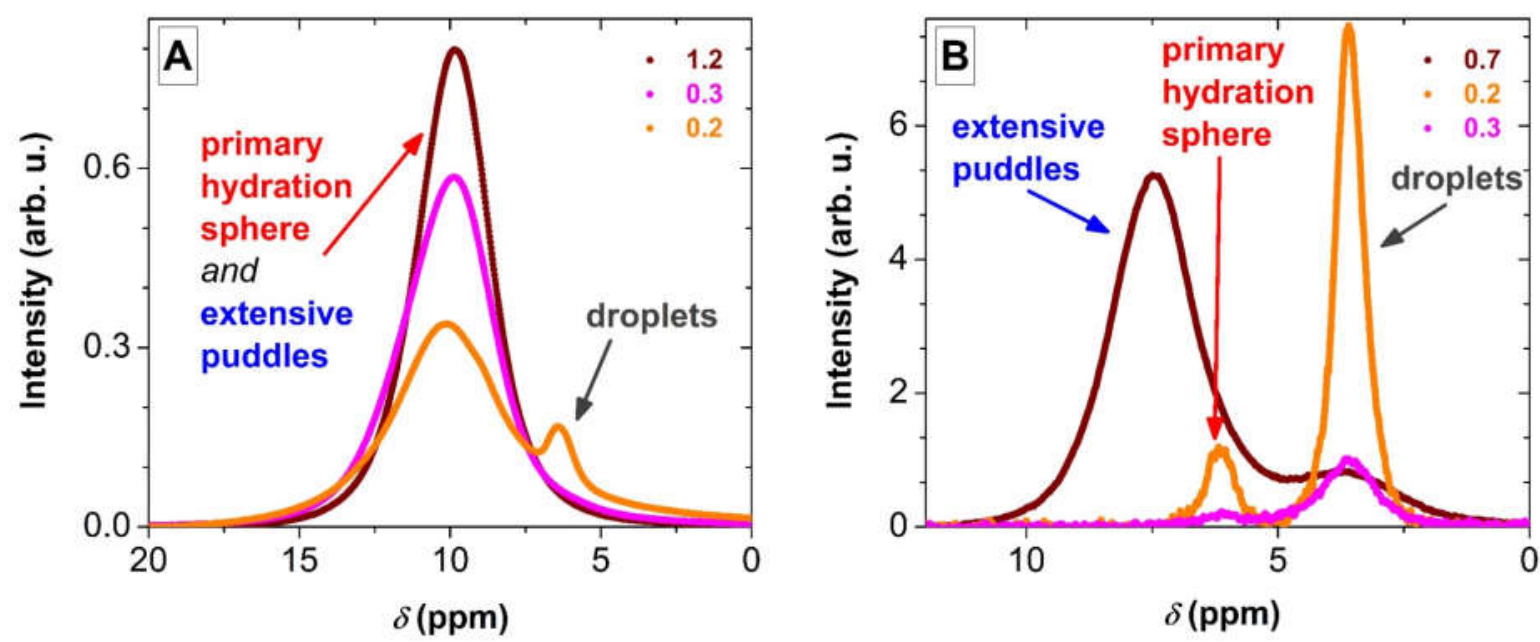

Figure S7. NMR spectra of hydrated CaAG recorded at different hydration levels, as given in the legend. The assignment of the signals is displayed on the figures using the same color code as in the case of NMR relaxometry (cf. Fig. 4). Panel A: ${ }^{1} \mathrm{H}$ NMR spectra. Panel B: PGSTE NMR spectra of diffusiometry experiments recorded using minimum gradient field strength, $\delta$ $=2 \mathrm{~ms}$ gradient pulse length and $\Delta=60 \mathrm{~ms}$ diffusion time.

Interestingly, the intensities of all PGSTE NMR peaks decrease significantly with the increase of the water content of the sample, and completely diminish at $0.4 \mathrm{~g} / \mathrm{g}$ water content 
(Fig. S7B). When the water content of the sample is increased further, a new well-defined peak appears in the PGSTE NMR spectrum with a minor shoulder at lower chemical shift, which is persistent even in the case of the well-hydrated samples. The key differences between the two types of NMR measurements are the total time of the experiment and the shifting of the magnetization of the sample. ${ }^{21-22}$ The ${ }^{1} \mathrm{H}$ NMR spectrum is recorded in ca. $0.01 \mathrm{~ms}$, while the PGSTE NMR spectrum is recorded in ca. $60 \mathrm{~ms}$. In the PGSTE NMR experiment, the sample is magnetized in the transversal plane only during the length of the gradient pulse ( $\delta=2 \mathrm{~ms})$, otherwise it is magnetized in the longitudinal plane. The $2 \mathrm{~ms}$ time is long enough for the transversal relaxation of those water protons that are in close association with the solid backbone of CaAG. This means that a $T_{2}$ filtering effect practically cancels the signal of the water protons in the primary hydration sphere in the PGSTE NMR spectra at higher water contents. Furthermore, the ca. $60 \mathrm{~ms}$ total timeframe of the PGSTE NMR experiment is long enough for the effective chemical exchange of water molecules localized in the different chemical environments, i.e. in the different relaxation domains of the sample.

The information content and the difference of the ${ }^{1} \mathrm{H}$ NMR and PGSTE NMR spectra can be interpreted based on the results of the NMR relaxometry experiments. Water is localized in hydrated $\mathrm{CaAG}$ as structural water associated to the macromolecules, in the primary hydration sphere of the Ca-alginate chains, and in droplets in the focal points of the porous network. Both the ${ }^{1} \mathrm{H}$ NMR spectrum and the PGSTE NMR spectrum of CaAG of $0.2 \mathrm{~g} / \mathrm{g}$ water content are structured. The major peak of the ${ }^{1} \mathrm{H}$ NMR spectra in Fig. S7A is assigned to water in the primary hydration sphere ( $2^{\text {nd }}$ relaxation domain), because structural water built into the solid backbone does not give a signal in liquid phase NMR spectroscopy. The minor peak at lower chemical shift in the ${ }^{1} \mathrm{H}$ NMR spectrum measured at $0.2 \mathrm{~g} / \mathrm{g}$ water content is assigned to small water droplets that form in the focal points of the solid pore network $\left(3^{\text {rd }}\right.$ relaxation domain). The peak at higher chemical shift is very small in the PGSTE NMR spectrum of the 
sample of $0.2 \mathrm{~g} / \mathrm{g}$ water content (Fig. S7B). The reason is that the transversal relaxation of the protons of water in the primary hydration sphere ( $2^{\text {nd }}$ relaxation domain $)$ is almost complete in $2 \mathrm{~ms}$. The relaxation of water is much slower in the droplets; thus these water molecules yield a signal in the PGSTE NMR spectrum at lower chemical shift. When the hydration level of CaAG increases, its ${ }^{1} \mathrm{H}$ NMR spectrum becomes unstructured, because the chemical shift values of the water molecules localized in the different structural regions are not significantly different. On the other hand, the PGSTE NMR spectrum remains structured even at high hydration levels, because the major signal of the water protons in the extensive primary hydration sphere is not present due to fast transversal relaxation ( $T_{2}$ filtering effect). Importantly, the chemical exchange of water within the ca. $60 \mathrm{~ms}$ timeframe of the PGSTE NMR measurement has a significant effect on the structure of the spectra, and causes the disappearance of the signal when the water content of the sample is between 0.4 and $0.6 \mathrm{~g} / \mathrm{g}$. Two counteracting effects are operational in shaping the PGSTE NMR spectra. One effect is the increase of the signal intensity, and the other effect is the increase of the rate of chemical exchange between low $T_{2}$ water in the primary hydration sphere and high $T_{2}$ water in the droplets by increasing hydration level. The fast exchange of water transfers the relaxation effect of the Ca-alginate backbone from the primary hydration sphere to the water droplets and causes the diminishing of the corresponding PGSTE NMR signal (transfer of $T_{2}$ filtering effect). The increase of the rate of water exchange and relaxation transfer dominantly shapes the spectra with the increase of the water content of $\mathrm{CaAG}$ up to ca. $0.6 \mathrm{~g} / \mathrm{g}$. At higher hydration levels, the effect of relaxation transfer is overcompensated by the increase of the water content and the increase of the $T_{2}$ value of the droplets. Thus, the intensity of the PGSTE NMR signal has a minimum as a function of the water content of CaAG. Finally, at water contents higher than $0.7 \mathrm{~g} / \mathrm{g}$, the PGSTE NMR spectrum is dominated by the signal of water molecules forming a quasi-bulk phase in the hydrogel-like Ca-alginate network. 


\section{References for Supporting Information}

(1) Ganesan, K.; Budtova, T.; Ratke, L.; Gurikov, P.; Baudron, V.; Preibisch, I.; Niemeyer, P.; Smirnova, I.; Milow, B. Review on the Production of Polysaccharide Aerogel Particles. Materials 2018, 11 (11), 2144, DOI: 10.3390/ma11112144.

(2) Preibisch, I.; Niemeyer, P.; Yusufoglu, Y.; Gurikov, P.; Milow, B.; Smirnova, I. PolysaccharideBased Aerogel Bead Production via Jet Cutting Method. Materials 2018, 11 (8), 1287, DOI: 10.3390/ma11081287.

(3) Mollica, G.; Ziarelli, F.; Lack, S.; Brunel, F.; Viel, S. Characterization of insoluble calcium alginates by solid-state NMR. Carbohydr. Polym. 2012, 87 (1), 383-391, DOI: 10.1016/j.carbpol.2011.07.066.

(4) Paul, G.; Bisio, C.; Braschi, I.; Cossi, M.; Gatti, G.; Gianotti, E.; Marchese, L. Combined solid-state NMR, FT-IR and computational studies on layered and porous materials. Chemical Society Reviews 2018, 47 (15), 5684-5739, DOI: 10.1039/C7CS00358G.

(5) Salomonsen, T.; Jensen, H. M.; Larsen, F. H.; Steuernagel, S.; Engelsen, S. B. Alginate monomer composition studied by solution- and solid-state NMR - A comparative chemometric study. Food Hydrocoll. 2009, 23 (6), 1579-1586, DOI: 10.1016/j.foodhyd.2008.11.009.

(6) Sperger, D. M.; Fu, S.; Block, L. H.; Munson, E. J. Analysis of composition, molecular weight, and water content variations in sodium alginate using solid-state NMR spectroscopy. J. Pharm. Sci. 2011, 100 (8), 3441-3452, DOI: $10.1002 / j p s .22559$.

(7) Maleki, H.; Montes, S.; Hayati-Roodbari, N.; Putz, F.; Huesing, N. Compressible, Thermally Insulating, and Fire Retardant Aerogels through Self-Assembling Silk Fibroin Biopolymers Inside a Silica Structure-An Approach towards 3D Printing of Aerogels. ACS Appl. Mater. Interfaces 2018, 10 (26), 22718-22730, DOI: 10.1021/acsami.8b05856.

(8) Diener, M.; Adamcik, J.; Sanchez-Ferrer, A.; Jaedig, F.; Schefer, L.; Mezzenga, R. Primary, Secondary, Tertiary and Quaternary Structure Levels in Linear Polysaccharides: From Random Coil, to Single Helix to Supramolecular Assembly. Biomacromolecules 2019, 20 (4), 1731-1739, DOI: 10.1021/acs.biomac.9b00087.

(9) Allen, S. G.; Stephenson, P. C. L.; Strange, J. H. Morphology of porous media studied by nuclear magnetic resonance. J. Chem. Phys. 1997, 106 (18), 7802-7809, DOI: Doi 10.1063/1.473780.

(10) Bardenhagen, I.; Dreher, W.; Fenske, D.; Wittstock, A.; Bäumer, M. Fluid distribution and pore wettability of monolithic carbon xerogels measured by $1 \mathrm{H}$ NMR relaxation. Carbon 2014, 68, 542-552, DOI: 10.1016/j.carbon.2013.11.033.

(11) Simina, M.; Nechifor, R.; Ardelean, I. Saturation-dependent nuclear magnetic resonance relaxation of fluids confined inside porous media with micrometer-sized pores. Magn. Reson. Chem. 2011, 49 (6), 314-9, DOI: $10.1002 / \mathrm{mrc} .2749$.

(12) Allen, S. G.; Stephenson, P. C. L.; Strange, J. H. Internal surfaces of porous media studied by nuclear magnetic resonance cryoporometry. J. Chem. Phys. 1998, 108 (19), 8195-8198, DOI: Doi $10.1063 / 1.476175$.

(13) Dore, J. C.; Webber, J. B. W.; Strange, J. H. Characterisation of porous solids using small-angle scattering and NMR cryoporometry. Colloid. Surface. A 2004, 241 (1-3), 191-200, DOI: 10.1016/j.colsurfa.2004.04.005.

(14) Petrov, O. V.; Furo, I. NMR cryoporometry: Principles, applications and potential. Prog. Nucl. Magn. Res. Spect. 2009, 54 (2), 97-122, DOI: 10.1016/j.pnmrs.2008.06.001.

(15) Gun'ko, V. M.; Turov, V. V.; Pakhlov, E. M.; Krupska, T. V.; Charmas, B. Effect of water content on the characteristics of hydro-compacted nanosilica. Appl. Surf. Sci. 2018, 459, 171-178, DOI: 10.1016/j.apsusc.2018.07.213. 
(16) Keri, M.; Nagy, Z.; Novak, L.; Szarvas, E.; Balogh, L. P.; Banyai, I. Beware of phosphate: evidence of specific dendrimer-phosphate interactions. Phys. Chem. Chem. Phys. 2017, 19 (18), 11540-11548, DOI: $10.1039 / \mathrm{c} 7 \mathrm{cp} 00875 \mathrm{a}$.

(17) Keri, M.; Peng, C.; Shi, X.; Banyai, I. NMR characterization of PAMAM_G5.NH2 entrapped atomic and molecular assemblies. J. Phys. Chem. B 2015, 119 (7), 3312-9, DOI: 10.1021/acs.jpcb.5b00272.

(18) Cho, C. H.; Hong, Y. S.; Kang, K.; Volkov, V. I.; Skirda, V.; Lee, C. Y.; Lee, C. H. Water selfdiffusion in Chlorella sp. studied by pulse field gradient NMR. Magn. Reson. Imaging 2003, 21 (9), 1009-17, DOI: 10.1016/S0730-725X(03)00206-6.

(19) Rottreau, T. J.; Parlett, C. M. A.; Lee, A. F.; Evans, R. Diffusion NMR Characterization of Catalytic Silica Supports: A Tortuous Path. J. Phys. Chem. C 2017, 121 (30), 16250-16256, DOI: 10.1021/acs.jpcc.7b02929.

(20) Valiullin, R.; Skirda, V. Time dependent self-diffusion coefficient of molecules in porous media. J. Chem. Phys. 2001, 114 (1), 452-458, DOI: Doi 10.1063/1.1328416.

(21) Cohen, Y.; Avram, L.; Frish, L. Diffusion NMR spectroscopy in supramolecular and combinatorial chemistry: an old parameter--new insights. Angew. Chem. Int. Ed. 2005, 44 (4), 520-54, DOI: 10.1002/anie.200300637.

(22) Isaacs, M. A.; Robinson, N.; Barbero, B.; Durndell, L. J.; Manayil, J. C.; Parlett, C. M. A.; D'Agostino, C.; Wilson, K.; Lee, A. F. Unravelling mass transport in hierarchically porous catalysts. J. Mater. Chem. A 2019, 7 (19), 11814-11825, DOI: 10.1039/c9ta01867k. 